\title{
Modulações epigenéticas da amamentação
}

- Modificações epigenéticas, como a atuação de microRNAs (RNAs não-codificantes), podem influenciar no status de saúde dos lactentes;

- Os microRNAs são sequências pequenas de nucleotídeos (entre 18 e 25) capazes de regular a expressão gênica;

- Mais de 1400 microRNAs já foram identificados em humanos;

- Os microRNAs podem ser encontrados em fluídos corporais, como o leite materno;

- Os microRNAs produzidos pela mãe podem ser transmitidos ao bebê durante a amamentação;

- A biodisponibilidade dos microRNAs do leite materno depende dos exossomos (estruturas responsáveis pelo transporte) e da integridade da mucosa intestinal do bebê;

- Os microRNAs presentes no leite materno, com destaque para o miRNA 148a, podem auxiliar na melhora da resposta imune dos bebês;

- Fórmulas infantis e leite de vaca possuem microRNAs, mas a biodisponibilidade é limitada e os estudos pouco conclusivos;

- O processo de pasteurização também pode limitar a biodisponibilidade dos microRNAs;

- Nessa perspectiva, surgiu a Nutrimirômica que estuda a influência da dieta nas modificações de expressão gênica capaz de envolver processos epigenéticos relacionados aos microRNAs e associados ao risco do desenvolvimento para doenças.

\section{Referências bibliográficas}

1. BALLARD O.; MORROW, A.L. Human milk composition: nutrients and bioactive factors. Pediatr Clin North Am; 60(1):49-74, 2013.

2. BURDGE, G.C.; LILLYCROP, K.A. Nutrition, epigenetics, and developmental plasticity: implications for understanding human disease. Annu Rev Nutr; 30:315-39, 2010.

3. GOLAN-GERSTL R, ELBAUM SHIFF Y, MOSHAYOFF V, SCHECTER D, LESHKOWITZ D, REIF S. Characterization and biological function of milkderived miRNAs. Mol Nutr Food Res; 61(10), 2017.

4. GRIFFITHS-JONES, S. miRBase: microRNA sequences and annotation. Curr Protoc Bioinformatics; Chapter 12:Unit 12.9.1-10, 2010.

5. MELNIK, B.C.; SCHMITZ, G. Milk's Role as an Epigenetic Regulator in Health and Disease. Diseases; 5(1). pii: E12, 2017.

6. QUINTANILHA, B.J.; REIS, B.Z.; DUARTE, G.B.S. et al. Nutrimiromics: Role of microRNAs and nutrition in Modulating Inflammation and Chronic Diseases. Nutrients; 9:1168, 2017.

7. TAMMEN, S.A.; FRISO, S.; CHOI, S.W. Epigenetics: the link between nature and nurture. Mol Aspects Med; 34(4):753-64, 2013.

8. VERDUCI, E.; BANDERALI, G.; BARBERI, S. et al. Epigenetic effects of human breast milk. Nutrients; 6(4):1711-24, 2014. 